$\underline{\text { Preprint typeset in JHEP style. - HYPER VERSION }}$

$\mathrm{DFF} / 350 / 03 / 00$

TTP00-06

\title{
Heavy Quark Impact Factor at Next-to-leading Level*
}

\author{
M. Ciafaloni \\ Dipartimento di Fisica, Università di Firenze and INFN, Sezione di Firenze, \\ Largo E. Fermi 2, I-50125 Firenze, Italy. Email: ciafaloni@fi.infn.it \\ G. Rodrigo \\ Institut für Teoretische Teilchenphysik, Universität Karlsruhe \\ D-76128 Karlsruhe, Germany. Email: rodrigo@particle.uni-karlsruhe.de
}

\begin{abstract}
We further analyze the definition and the calculation of the heavy quark impact factor at next-to-leading (NL) $\log s$ level, and we provide its analytical expression in a previously proposed $\boldsymbol{k}$-factorization scheme. Our results indicate that $\boldsymbol{k}$-factorization holds at NL level with a properly chosen energy scale, and with the same gluonic Green's function previously found in the massless probe case.
\end{abstract}

KEYwords: Heavy quarks, small-x, NLO corrections.

\footnotetext{
${ }^{*}$ Work supported in part by the E.U. QCDNET contract FMRX-CT98-0194 and by MURST (Italy).
} 


\section{Introduction}

Recent improvements [1] of the next-to-leading $\log x$ (NLx) results [2] in the BFKL framework, have stabilized the small-x behaviour in QCD, so that a phenomenological analysis of deep inelastic processes (DIS) seems now possible.

However, both the gluon density (satisfying the improved equation) and the impact factors are needed in order to use $\boldsymbol{k}$-factorization (Sec. 2) to compute DIS or double DIS processes. So far, NL $x$ impact factors have been found for the unphysical case of massless initial quarks and gluons only [3, [1. Partial features for massive quarks [5] and for colourless sources [6] are known too.

In this paper we derive complete results for the case of initial massive quarks, with a twofold purpose. First, we want to check the validity of the $\boldsymbol{k}$-factorization scheme introduced in Ref. [4], or, in other words, to derive the same gluon Green's function with an explicit massive quark impact factor which satisfies the expected collinear properties. Secondly, we develop as a byproduct some analytical techniques which are needed to deal with two-scale problems, which are hopefully useful to cope with the physical cases also.

The results of the paper rest on two observations. The first one, motivated in Sec. 3, is that the factorized scale relevant in a high-energy two-scale process coupled to heavy quarks is $s_{0}=\operatorname{Max}\left(k_{1}, m_{1}\right) \operatorname{Max}\left(k_{2}, m_{2}\right)$, where $k_{1}$ and $k_{2}$ denote the relevant gluon virtualities, rather than $s_{0}=k_{1} k_{2}$, as in the massless quark case. In fact, by subtracting the kernel contribution with such a scale we are able in Sec. 4.2 to derive a result for the massive quark impact factor which is finite for $s \rightarrow \infty$, and has all the desired properties.

The second observation is that we are able to disentangle the $(\mathrm{m} / \mathrm{k})$-dependence of the impact factor by explicitly computing its Mellin transform and its inverse. Given the singular energy dependence of the squared amplitude and of the phase space in the intermediate steps, this is by no means a trivial result and requires a careful handling of Mellin transform integrals in dimensional regularization, as explained in Sec. 4.1.

The outcome of such analysis is that the NLx constant $H$-kernels, previously introduced in the gluon Green's function [4], are indeed probe-independent, and that the ensuing impact factors only contain factorizable single logarithmic collinear singularities. The use of such information in the improved small- $x$ equation and the left-over problems are discussed in Sec. 5 .

\section{2. k-Factorization in dijet production}

We consider the high-energy scattering of two partons a and $\mathrm{b}$ with momenta $p_{1}$

and $p_{2}$ respectively. Following [3], the colour averaged differential cross section is factorized in a gauge-invariant way into a Green's function $\mathcal{G}_{\omega}$ and impact factors $h_{\mathrm{a}}$ 
and $h_{\mathrm{b}}$ (Fig. 1)

$$
\frac{\mathrm{d} \sigma_{\mathrm{ab}}}{\mathrm{d}\left[\boldsymbol{k}_{1}\right] \mathrm{d}\left[\boldsymbol{k}_{2}\right]}=\int \frac{\mathrm{d} \omega}{2 \pi i \omega} h_{\mathrm{a}}\left(\boldsymbol{k}_{1}\right) \mathcal{G}_{\omega}\left(\boldsymbol{k}_{1}, \boldsymbol{k}_{2}\right) h_{\mathrm{b}}\left(\boldsymbol{k}_{2}\right)\left(\frac{s}{s_{0}\left(\boldsymbol{k}_{1}, \boldsymbol{k}_{2}\right)}\right)^{\omega} .
$$

We adopt $\mathrm{d}[\boldsymbol{k}]=\mathrm{d}^{2+2 \varepsilon} \boldsymbol{k} / \pi^{1+\varepsilon}$ as transverse space measure. The transverse plane is defined with respect to the incoming momenta $p_{1}$ and $p_{2}$. The transverse momenta $\boldsymbol{k}_{1}$ and $\boldsymbol{k}_{2}$ play the role of hard scales of the process. By definition, the impact factors are free of high-energy gluon exchanges, which are subtracted out, but can still contain collinear singularities which need to be factored out. The Green's function $\mathcal{G}_{\omega}$ incorporates all the Regge-gluon exchanges between the two partons. The energyscale $s_{0}\left(\boldsymbol{k}_{1}, \boldsymbol{k}_{2}\right)$ will be chosen later on.

At the next-to-leading $\log x$ (NLx) accuracy the Green's function $\mathcal{G}_{\omega}$ has the following general form

$$
\mathcal{G}_{\omega}=\left(1+\bar{\alpha}_{s} H_{L}\right)\left[1-\frac{\bar{\alpha}_{s}}{\omega}\left(K_{0}+K_{N L}\right)\right]^{-1}\left(1+\bar{\alpha}_{s} H_{R}\right),
$$

where $K_{0}$ and $K_{N L}$ are the leading $\log x$ (Lx) and the NLx BFKL kernels [2] respectively, $H_{R}\left(H_{L}\right)$ are operator factors introduced in [1] and

$$
\bar{\alpha}_{s}=\frac{\alpha_{s} N_{c}}{\pi}, \quad \alpha_{s}=\frac{g^{2} \Gamma(1-\varepsilon) \mu^{2 \varepsilon}}{(4 \pi)^{1+\varepsilon}},
$$

is the dimensionless strong coupling constant.

As explained in [4], the identification of the second order impact factors, $h_{\mathrm{a}}^{(1)}$ and $h_{\mathrm{b}}^{(1)}$, is affected by a double factorization scheme ambiguity, due to both the choice of the scale $s_{0}$ and of the kernels $H_{R}\left(H_{L}\right)$. The latter were introduced by Ciafaloni and Colferai (CC) in [4] Figure 1: Diagrammatic representaso as to provide partonic impact factors free of tion of $\boldsymbol{k}$-factorization. double log collinear divergences for the factor-

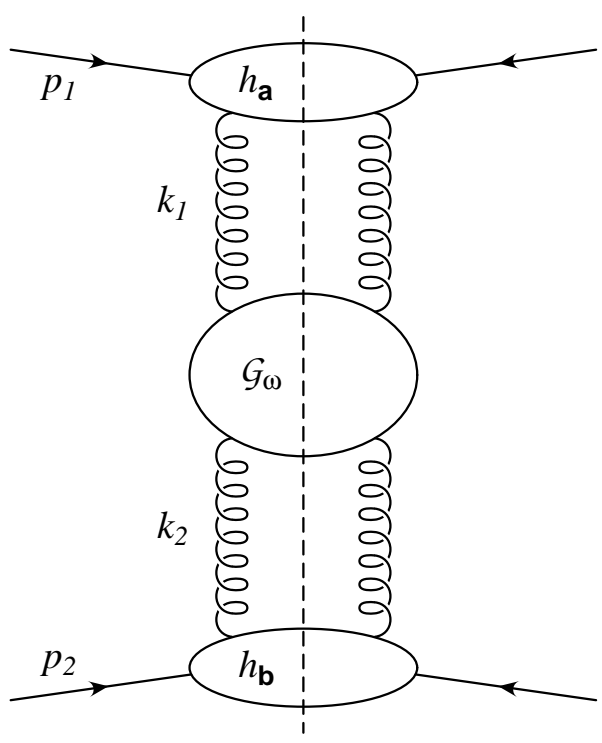
ized scale choice $s_{0}=k_{1} k_{2}$. It was also shown that the left-over single logarithmic divergences could be factorized by the usual DGLAP approach. A different factorization scheme, allowing double logarithmic divergences, was used instead in [5], where an integral representation for the massive quark impact factor was presented also.

In this paper we extend the $\mathrm{CC}$ scheme to the massive quark case, by showing that the gluonic Green's function stays the same and that the collinear divergences of the massive impact factor stay single logarithmic too. Although the use of the $H$ 
kernels is optional for colourless sources [6], for which they can be incorporated in the impact factors, we think that they help in stabilizing the collinear behaviour of the gluonic Green's function, as already noticed in [3].

In the following we use the notation $p_{1}, p_{2}\left(\lambda_{1}, \lambda_{2}\right)$ for the initial parton's momenta (helicities) and the indices 3,4 (possibly 5 ) for the final ones, with the Sudakov parametrization

$$
\begin{aligned}
& k_{1}=p_{1}-p_{3}=z_{1} \bar{p}_{1}-\frac{\boldsymbol{k}_{1}^{2}}{\left(1-z_{1}\right) s} \bar{p}_{2}+k_{1 \perp}, \\
& k_{2}=p_{2}-p_{4}=-\frac{\boldsymbol{k}_{2}^{2}}{\left(1-z_{2}\right) s} \bar{p}_{1}+z_{2} \bar{p}_{2}+k_{2 \perp},
\end{aligned}
$$

where we have introduced Sudakov variables $z_{i}$ and transverse spacelike vectors $k_{i \perp}$ perpendicular to the plane of the initial particle momenta light-cone basis $\left\langle\bar{p}_{1}, \bar{p}_{2}\right\rangle$

$$
p_{1}=\bar{p}_{1}+\frac{m_{1}^{2}}{s} \bar{p}_{2}, \quad p_{2}=\bar{p}_{2}+\frac{m_{2}^{2}}{s} \bar{p}_{1}
$$

$\bar{p}_{i} \cdot k_{j \perp}=0, \bar{p}_{i}^{2}=0, p_{i}^{2}=m_{i}^{2}$, with $D-2$ Euclidean components $\boldsymbol{k}_{i}: \boldsymbol{k}_{i}^{2}=-k_{i \perp}^{2}>0$. We also define $\boldsymbol{q}=\boldsymbol{k}_{1}+\boldsymbol{k}_{2}$ as the transverse momentum of parton 5. For simplicity, we use in the sequel $q=|\boldsymbol{q}|, k_{i}=\left|\boldsymbol{k}_{i}\right|$ and we consider parton b as massless, $m_{2}=0$, $m_{1}=m$.

\section{Factorization scheme and calculational procedure}

Let's consider first the high-energy scattering of two partons $\mathrm{a}$ and $\mathrm{b}$ where $\mathrm{a}=\mathrm{q}$ is $\mathrm{a}$ heavy quark of mass $m$ with real emission of an extra gluon $g$ that we assume in the heavy quark fragmentation region (Fig. 2). In terms of invariants, we work in the kinematical region $s_{2}=\left(q+p_{4}\right)^{2} \simeq z_{1} s \gg s_{1}=\left(p_{3}+q\right)^{2} \simeq$ $q^{2} / z_{1}$, so that $z_{1}>q / \sqrt{s}$ is the fragmentation phase space boundary. The Born differential cross section in this high energy region can be calculated in a straightforward way (e.g., by eikonal coupling to the incoming parton $b$ ),

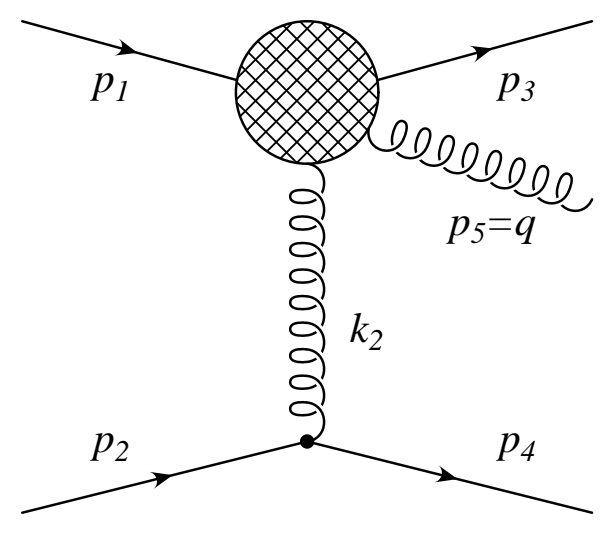

Figure 2: Real gluon emission in the fragmentation region of quark a. 
and results to be

$$
\begin{gathered}
\frac{\mathrm{d} \sigma_{\mathrm{qgb}}}{\mathrm{d} z_{1} \mathrm{~d}\left[\boldsymbol{k}_{1}\right] \mathrm{d}\left[\boldsymbol{k}_{2}\right]}=A_{\varepsilon} h_{\mathrm{b}}^{(0)}\left(\boldsymbol{k}_{2}\right) \\
\times \frac{1}{N_{c}}\left[C _ { F } \left(\mathcal{P}_{\mathrm{gq}}\left(z_{1}, \varepsilon\right) \frac{z_{1}^{2}}{\left[\boldsymbol{q}^{2}+m^{2} z_{1}^{2}\right]\left[\left(\boldsymbol{q}-z_{1} \boldsymbol{k}_{2}\right)^{2}+m^{2} z_{1}^{2}\right]}\right.\right. \\
\left.-\frac{m^{2}}{\boldsymbol{k}_{2}^{2}} \frac{z_{1}^{3}\left(1-z_{1}\right)\left[\left(2 \boldsymbol{q}-z_{1} \boldsymbol{k}_{2}\right) \cdot \boldsymbol{k}_{2}\right]^{2}}{\left[\boldsymbol{q}^{2}+m^{2} z_{1}^{2}\right]^{2}\left[\left(\boldsymbol{q}-z_{1} \boldsymbol{k}_{2}\right)^{2}+m^{2} z_{1}^{2}\right]^{2}}\right) \\
+N_{c}\left(\mathcal{P}_{\mathrm{gq}}\left(z_{1}, \varepsilon\right) \frac{\left(1-z_{1}\right)\left[\boldsymbol{q} \cdot\left(\boldsymbol{q}-z_{1} \boldsymbol{k}_{2}\right)+m^{2} z_{1}^{2}\right]}{\left[\boldsymbol{k}_{1}^{2}+m^{2} z_{1}^{2}\right]\left[\boldsymbol{q}^{2}+m^{2} z_{1}^{2}\right]\left[\left(\boldsymbol{q}-z_{1} \boldsymbol{k}_{2}\right)^{2}+m^{2} z_{1}^{2}\right]}\right. \\
\left.\left.-\frac{m^{2}}{\boldsymbol{k}_{2}^{2}} \frac{z_{1}\left(1-z_{1}\right)^{2} \boldsymbol{k}_{2} \cdot\left(2 \boldsymbol{q}-\boldsymbol{k}_{2}\right)\left[\boldsymbol{k}_{2} \cdot\left(2 \boldsymbol{q}-\boldsymbol{k}_{2}\right)-z_{1} \boldsymbol{k}_{2}^{2}\right]}{\left[\boldsymbol{k}_{1}^{2}+m^{2} z_{1}^{2}\right]^{2}\left[\boldsymbol{q}^{2}+m^{2} z_{1}^{2}\right]\left[\left(\boldsymbol{q}-z_{1} \boldsymbol{k}_{2}\right)^{2}+m^{2} z_{1}^{2}\right]}\right)\right],
\end{gathered}
$$

where

$$
\mathcal{P}_{\mathrm{gq}}\left(z_{1}, \varepsilon\right)=\frac{1}{2 z_{1}}\left[1+\left(1-z_{1}\right)^{2}+\varepsilon z_{1}^{2}\right],
$$

is related to the quark to gluon splitting function,

$$
h^{(0)}(\boldsymbol{k})=\sqrt{\frac{\pi}{N_{c}^{2}-1}} \frac{2 C_{F} \alpha_{s} N_{\varepsilon}}{\boldsymbol{k}^{2} \mu^{2 \varepsilon}}, \quad N_{\varepsilon}=\frac{(4 \pi)^{\varepsilon / 2}}{\Gamma(1-\varepsilon)},
$$

is the leading order impact factor, being the same for quarks and gluons, $\mu$ is the renormalization scale, and

$$
A_{\varepsilon}=\boldsymbol{k}^{2} h^{(0)}(\boldsymbol{k}) \frac{\bar{\alpha}_{s}}{\Gamma(1-\varepsilon) \mu^{2 \varepsilon}},
$$

is a constant that contains the dependence on the strong coupling constant and some colour factors.

Though complicated at first sight, eq.(3.1) has some simple features that we now comment upon. First of all, only the $N_{c}$ part is really relevant to our purposes, the $C_{F}$ part being canceled with virtual correction upon $z_{1}$ and $\boldsymbol{k}_{1}$ integration (see the following). We consider

$$
\begin{aligned}
& \left.\frac{\mathrm{d} \sigma_{\mathrm{qgb}}}{\mathrm{d} z_{1} \mathrm{~d}\left[\boldsymbol{k}_{1}\right] \mathrm{d}\left[\boldsymbol{k}_{2}\right]}\right|_{N_{c}}=A_{\varepsilon} h_{\mathrm{b}}^{(0)}\left(\boldsymbol{k}_{2}\right) \\
\times & {\left[\mathcal{P}_{\mathrm{gq}}\left(z_{1}, \varepsilon\right) \frac{\left(1-z_{1}\right)\left[\boldsymbol{q} \cdot\left(\boldsymbol{q}-z_{1} \boldsymbol{k}_{2}\right)+m^{2} z_{1}^{2}\right]}{\left[\boldsymbol{k}_{1}^{2}+m^{2} z_{1}^{2}\right]\left[\boldsymbol{q}^{2}+m^{2} z_{1}^{2}\right]\left[\left(\boldsymbol{q}-z_{1} \boldsymbol{k}_{2}\right)^{2}+m^{2} z_{1}^{2}\right]}\right.} \\
& \left.-\frac{m^{2}}{\boldsymbol{k}_{2}^{2}} \frac{z_{1}\left(1-z_{1}\right)^{2} \boldsymbol{k}_{2} \cdot\left(2 \boldsymbol{q}-\boldsymbol{k}_{2}\right)\left[\boldsymbol{k}_{2} \cdot\left(2 \boldsymbol{q}-\boldsymbol{k}_{2}\right)-z_{1} \boldsymbol{k}_{2}^{2}\right]}{\left[\boldsymbol{k}_{1}^{2}+m^{2} z_{1}^{2}\right]^{2}\left[\boldsymbol{q}^{2}+m^{2} z_{1}^{2}\right]\left[\left(\boldsymbol{q}-z_{1} \boldsymbol{k}_{2}\right)^{2}+m^{2} z_{1}^{2}\right]}\right],
\end{aligned}
$$

The latter expression reduces, as expected to the known [4] result for $m \rightarrow 0$, and matches the $\mathrm{L} x$ differential cross section

$$
\frac{\mathrm{d} \sigma_{\mathrm{qgb}}^{(L)}}{\mathrm{d} z_{1} \mathrm{~d}\left[\boldsymbol{k}_{1}\right] \mathrm{d}\left[\boldsymbol{k}_{2}\right]}=h_{\mathrm{q}}^{(0)}\left(\boldsymbol{k}_{1}\right) h_{\mathrm{b}}^{(0)}\left(\boldsymbol{k}_{2}\right) \frac{\bar{\alpha}_{s}}{\boldsymbol{q}^{2} \Gamma(1-\varepsilon) \mu^{2 \varepsilon}} \frac{1}{z_{1}},
$$


in the limit $z_{1} \rightarrow$ 0. However, for eq.(3.6) to be a good approximation to eq.(3.5), we should require

$$
z_{1} \ll q / k_{1}, \quad z_{1} \ll q / m, \quad z_{1} \ll k_{1} / m
$$

The first two cutoffs can be summarized by $z_{1}<q / \operatorname{Max}\left(k_{1}, m\right)$, which is a coherence condition for the case of heavy quarks, saying that the rapidity of the gluon cannot exceed that of the final quark. By integrating the leading expression (3.6) with the constraints (3.7) in the fragmentation region $z_{1}>q / \sqrt{s}$, we obtain

$$
\frac{1}{h_{\mathrm{b}}^{(0)}\left(\boldsymbol{k}_{2}\right)} \frac{\mathrm{d} \sigma_{\mathrm{qgb}}^{(L)}}{\mathrm{d}\left[\boldsymbol{k}_{1}\right] \mathrm{d}\left[\boldsymbol{k}_{2}\right]}=\frac{\bar{\alpha}_{s} h_{\mathrm{q}}^{(0)}\left(\boldsymbol{k}_{1}\right)}{\boldsymbol{q}^{2} \Gamma(1-\varepsilon) \mu^{2 \varepsilon}}\left(\log \frac{\sqrt{s}}{\operatorname{Max}\left(k_{1}, m\right)}-\log \frac{q}{k_{1}} \Theta_{q k_{1}}\right) \text {. }
$$

This expression is an estimate of the leading contribution contained in the complete result eq.(3.1), which should be subtracted out in order to yield the impact factor in the massive quark case.

Compared to the subtraction (or factorization) scheme adopted in [4] for $m=0$, the expression (3.8) differs by the replacement $k_{1} \rightarrow \operatorname{Max}\left(k_{1}, m\right)$, which leads, by adding the symmetrical fragmentation region, to the choice for the factorized scale in eq. (2.1)

$$
s_{0}=\operatorname{Max}\left(k_{1}, m_{1}\right) \operatorname{Max}\left(k_{2}, m_{2}\right),
$$

$m_{1}$ being the mass of quark a and $m_{2}$ the mass of quark $b$.

By considering now both real and virtual contributions to the fragmentation function $F_{\mathrm{q}}\left(z_{1}, \boldsymbol{k}_{1}, \boldsymbol{k}_{2}\right)$, we are led to introduce the following definition of the impact factor $h_{\mathrm{q}}^{(1)}(\boldsymbol{k})$ :

$$
\begin{aligned}
& \int_{q / \sqrt{s}}^{1} \mathrm{~d} z_{1} \int \mathrm{d}\left[\boldsymbol{k}_{1}\right] F_{\mathbf{q}}\left(z_{1}, \boldsymbol{k}_{1}, \boldsymbol{k}_{2}\right) \\
& =\int \mathrm{d}\left[\boldsymbol{k}_{1}\right] \bar{\alpha}_{s} h_{\mathbf{q}}^{(0)}\left(\boldsymbol{k}_{1}\right) K_{0}\left(\boldsymbol{k}_{1}, \boldsymbol{k}_{2}\right)\left(\log \frac{\sqrt{s}}{\operatorname{Max}\left(k_{1}, m\right)}-\log \frac{q}{k_{1}} \Theta_{q k_{1}}\right)+h_{\mathbf{q}}^{(1)}\left(\boldsymbol{k}_{2}\right)
\end{aligned}
$$

where

$$
\bar{\alpha}_{s} K_{0}\left(\boldsymbol{k}_{1}, \boldsymbol{k}_{2}\right)=\frac{\bar{\alpha}_{s}}{\boldsymbol{q}^{2} \Gamma(1-\varepsilon) \mu^{2 \varepsilon}}+2 \omega^{(1)}\left(\boldsymbol{k}_{1}^{2}\right) \delta[\boldsymbol{q}], \quad \delta[\boldsymbol{q}]=\pi^{1+\varepsilon} \delta^{2+2 \varepsilon}(\boldsymbol{q})
$$

is the leading kernel, with

$$
\omega^{(1)}\left(\boldsymbol{k}^{2}\right)=-\frac{g^{2} N_{c} \boldsymbol{k}^{2}}{(4 \pi)^{2+\varepsilon}} \int \frac{\mathrm{d}[\boldsymbol{p}]}{\boldsymbol{p}^{2}(\boldsymbol{k}-\boldsymbol{p})^{2}}=-\frac{\bar{\alpha}_{s}}{2 \varepsilon} \frac{\Gamma^{2}(1+\varepsilon)}{\Gamma(1+2 \varepsilon)}\left(\frac{\boldsymbol{k}^{2}}{\mu^{2}}\right)^{\varepsilon},
$$


the gluon Regge trajectory. Eq.(3.10) reduces for $m=0$, to the definition adopted in Ref. [4], and in particular contains the subtraction term $\log q / k_{1} \Theta_{q k_{1}}$ which provides the expression $\left(H_{R}=H_{L}^{\dagger}=H\right)$

$$
H\left(\boldsymbol{k}_{1}, \boldsymbol{k}_{2}\right)=-\frac{1}{\boldsymbol{q}^{2} \Gamma(1-\varepsilon) \mu^{2 \varepsilon}} \log \frac{q}{k_{1}} \Theta_{q k_{1}},
$$

for the $H$ kernel in the $\boldsymbol{k}$-factorization formula.

In order to simplify our subsequent calculations, we shall then use the known result [4] for $m=0$

$$
\begin{aligned}
h_{\mathbf{q}, m=0}^{(1)}\left(\boldsymbol{k}_{2}\right) & =h_{\mathbf{q}}^{(0)}\left(\boldsymbol{k}_{2}\right) \omega^{(1)}\left(\boldsymbol{k}_{2}^{2}\right)\left[\left(\frac{11}{6}-\frac{n_{f}}{3 N_{c}}\right)+\left(\frac{3}{2}-\frac{1}{2} \varepsilon\right)\right. \\
& \left.-\left(\frac{67}{18}-\frac{\pi^{2}}{6}-\frac{5 n_{f}}{9 N_{c}}\right) \varepsilon\right],
\end{aligned}
$$

and we shall explicitly compute only the difference for a non vanishing mass. For this reason, we write the fragmentation vertex for a heavy quark as the massless fragmentation vertex plus an extra quark mass dependent contribution that cancels out for $m=0$

$$
F_{\mathrm{q}}\left(z_{1}, \boldsymbol{k}_{1}, \boldsymbol{k}_{2}\right)=F_{\mathrm{q}}^{m=0}\left(z_{1}, \boldsymbol{k}_{1}, \boldsymbol{k}_{2}\right)+\Delta F_{\mathrm{q}}\left(z_{1}, \boldsymbol{k}_{1}, \boldsymbol{k}_{2}\right) .
$$

Then, we find the following relationship between the massless quark impact factor and the heavy quark impact factor

$$
\begin{gathered}
h_{\mathbf{q}}^{(1)}\left(\boldsymbol{k}_{2}\right)=h_{\mathbf{q}, m=0}^{(1)}\left(\boldsymbol{k}_{2}\right)+\int_{0}^{1} \mathrm{~d} z_{1} \int \mathrm{d}\left[\boldsymbol{k}_{1}\right] \Delta F_{\mathbf{q}}\left(z_{1}, \boldsymbol{k}_{1}, \boldsymbol{k}_{2}\right) \\
+\int \mathrm{d}\left[\boldsymbol{k}_{1}\right] \bar{\alpha}_{s} h_{\mathbf{q}}^{(0)}\left(\boldsymbol{k}_{1}\right) K_{0}\left(\boldsymbol{k}_{1}, \boldsymbol{k}_{2}\right) \log \frac{m}{k_{1}} \Theta_{m k_{1}} .
\end{gathered}
$$

The most complicated integral that remains in the r.h.s. of eq.(3.16) is the one for $\Delta F_{\mathrm{q}}$ which will be calculated through its Mellin transform in $\boldsymbol{k}_{2}$. Notice also that the integration limits in $z_{1}$ have been extended down to $z_{1}=0$. Since $\Delta F_{\mathrm{q}}$ is regular at $z_{1}=0$ this change introduces only a negligible error of order $1 / s$.

\section{Mellin transform and its inverse}

In order to perform the calculation outlined in eq.(3.16), we proceed in two steps. First, we perform analytically the $\boldsymbol{k}_{1}$ integration of eq.(3.1) by reducing the $\boldsymbol{k}_{1}$ integrals to two denominators, as explained in detail in Appendix A. Then, we consider the virtual contributions [7] quoted in Appendix B, and we organize them in terms of momentum fraction integrals only. Finally, summing up real and virtual 
contributions to the fragmentation vertex (see eq.(A.6) and eq.(B.6) at the appendices) (helicity non conserving not included) we obtain the following expression for the difference $\Delta F_{\mathrm{q}}\left(\boldsymbol{k}_{2}\right)$, arising from the second term in the r.h.s. of eq.(3.16)

$$
\begin{aligned}
& \Delta F_{\mathrm{q}}\left(\boldsymbol{k}_{2}\right)=\Delta F_{\mathrm{q}, \text { real }}\left(\boldsymbol{k}_{2}\right)+\Delta F_{\mathrm{q}, \text { virt }}\left(\boldsymbol{k}_{2}\right)=A_{\varepsilon}\left[\frac{\Gamma(-\varepsilon)}{2(1+2 \varepsilon)} \frac{\left(m^{2}\right)^{\varepsilon}}{\boldsymbol{k}_{2}^{2}}\right. \\
& +\frac{\Gamma(1-\varepsilon)}{2}\left\{\int_{0}^{1} \int_{0}^{1} \mathrm{~d} z_{1} \mathrm{~d} x\left(\frac{1-z_{1}}{z_{1}}+\frac{1+\varepsilon}{2} z_{1}\right)\right. \\
& \quad \times\left[\frac{1}{\left[x(1-x) \boldsymbol{k}_{2}^{2}+m^{2} z_{1}^{2}\right]^{1-\varepsilon}}-\frac{1}{\left[x(1-x) \boldsymbol{k}_{2}^{2}\right]^{1-\varepsilon}}\right] \\
& \left.\left.+\frac{2 m^{2}}{\boldsymbol{k}_{2}^{2}} \int_{0}^{1} \int_{0}^{1} \frac{z_{1}\left(1-z_{1}\right) \mathrm{d} z_{1} \mathrm{~d} x}{\left[x(1-x) \boldsymbol{k}_{2}^{2}+m^{2} z_{1}^{2}\right]^{1-\varepsilon}}\right\}\right],
\end{aligned}
$$

where in $\Delta \Gamma_{\mathrm{qq}}^{(+)}\left(\boldsymbol{k}_{2}\right)$, eq. (B.5) of Appendix B), the integration variable $x$ has been identified with $z_{1}$ to simplify the sum. Note again that, because of the subtraction of the $m=0$ part, the $z_{1}$-integrals are convergent at $z_{1}=0$.

\subsection{Mellin integrals}

To perform the last integrations in eq.(4.1) we first calculate its Mellin transform

$$
\Delta \tilde{F}_{\mathbf{q}}(\gamma)=\Gamma(1+\varepsilon)\left(m^{2}\right)^{-\varepsilon} \int \mathrm{d}\left[\boldsymbol{k}_{2}\right]\left(\frac{\boldsymbol{k}_{2}^{2}}{m^{2}}\right)^{\gamma-1} \Delta F_{\mathrm{q}}\left(\boldsymbol{k}_{2}\right),
$$

yielding

$$
\begin{aligned}
& \Delta \tilde{F}_{\mathbf{q}}(\gamma)=A_{\varepsilon}\left(m^{2}\right)^{\varepsilon} \frac{\Gamma(\gamma+\varepsilon) \Gamma(1-\gamma-2 \varepsilon) \Gamma^{2}(1-\gamma-\varepsilon)}{8 \Gamma(2-2 \gamma-2 \varepsilon)} \\
& \times\left[\frac{1+\varepsilon}{\gamma+2 \varepsilon}+\frac{2}{1-2 \gamma-4 \varepsilon}\left(\frac{1}{1-\gamma-2 \varepsilon}-\frac{1}{3-2 \gamma-2 \varepsilon}\right)\right] .
\end{aligned}
$$

Although this Mellin transform is finite for $\varepsilon \rightarrow 0$ the limit $\varepsilon=0$ cannot be taken in this expression. In fact, it is straightforward, though not trivial, to show that the r.h.s. of eq.(4.1) behaves as

$$
\Delta F_{\mathrm{q}}\left(\boldsymbol{k}_{2}\right) \underset{k 2 \ll m}{\simeq}\left(\boldsymbol{k}_{2}^{2}\right)^{\varepsilon-1}, \quad \Delta F_{\mathrm{q}}\left(\boldsymbol{k}_{2}\right) \underset{k 2 \gg m}{\simeq}\left(\boldsymbol{k}_{2}^{2}\right)^{-1}\left(m^{2}\right)^{\varepsilon} .
$$

Therefore, the Mellin transform converges only in the small band $1-2 \varepsilon<R e \gamma<1-\varepsilon$ and the $\varepsilon$ dependence should be kept until the end.

To recover $\Delta F_{\mathrm{q}}\left(\boldsymbol{k}_{2}\right)$ we should calculate the following inverse Mellin transform

$$
\Delta F_{\mathrm{q}}\left(\boldsymbol{k}_{2}\right)=\frac{1}{m^{2}} \int_{1-2 \varepsilon<\operatorname{Re\gamma }<1-\varepsilon} \frac{\mathrm{d} \gamma}{2 \pi i}\left(\frac{\boldsymbol{k}_{2}^{2}}{m^{2}}\right)^{-\gamma-\varepsilon} \Delta \tilde{F}_{\mathrm{q}}(\gamma) .
$$


We consider first the limit $\boldsymbol{k}_{2}^{2}>m^{2}$. Then, we displace the integration contour around the positive real semiaxis, enclosing all the poles placed in $\gamma \geq 1-\varepsilon$, the smaller one giving the smaller power in $m / k_{2}$. The first pole, at $\gamma=1-\varepsilon$, yields the following result

$$
\Delta F_{\mathrm{q}}\left(\boldsymbol{k}_{2}\right)=\bar{\alpha}_{s} h_{\mathrm{q}}^{(0)}\left(\boldsymbol{k}_{2}\right)\left\{-\frac{2+3 \varepsilon+2 \varepsilon^{2}}{4 \varepsilon^{2}(1+2 \varepsilon)}\left(\frac{m^{2}}{\mu^{2}}\right)^{\varepsilon}+\mathcal{O}\left(m / k_{2}\right)\right\} .
$$

For $\boldsymbol{k}_{2}^{2}<m^{2}$ we displace the integration contour around the negative real semiaxis, enclosing all the poles placed in $\gamma \leq 1-2 \varepsilon$. As before, the first pole, at $\gamma=1-2 \varepsilon$, gives the answer at order $\mathcal{O}\left(k_{2} / m\right)$

$$
\begin{aligned}
\Delta F_{\mathrm{q}}\left(\boldsymbol{k}_{2}\right) & =h_{\mathrm{q}}^{(0)}\left(\boldsymbol{k}_{2}\right)\left\{\omega ^ { ( 1 ) } ( \boldsymbol { k } _ { 2 } ^ { 2 } ) \left[-\frac{1+5 \varepsilon-2 \varepsilon^{2}}{2(1+2 \varepsilon)}-\log \left(\frac{\boldsymbol{k}_{2}^{2}}{m^{2}}\right)\right.\right. \\
& \left.+\psi(1-\varepsilon)-\psi(1)-2 \psi(\varepsilon)+2 \psi(2 \varepsilon)]+\mathcal{O}\left(k_{2} / m\right)\right\} .
\end{aligned}
$$

Since $\omega^{(1)} \sim 1 / \varepsilon$ is infrared singular (cf. eq.(3.12) ), both formulas show double logarithmic singularities of type $1 / \varepsilon^{2}$ and $1 / \varepsilon \log \left(\boldsymbol{k}_{2}^{2} / m^{2}\right)$.

The last ingredient we need in order to extract the next-to-leading quark impact factor is the last term in the r.h.s. of eq.(3.16). For the real emission contribution to $K_{0}$ we get the integral

$$
I_{m}=\int \mathrm{d}\left[\boldsymbol{k}_{1}\right] \frac{\bar{\alpha}_{s} h_{\mathbf{q}}^{(0)}\left(\boldsymbol{k}_{1}\right)}{\boldsymbol{q}^{2} \Gamma(1-\varepsilon) \mu^{2 \varepsilon}} \log \frac{m}{k_{1}} \Theta_{m k_{1}} .
$$

We use the following representation

$$
\log \frac{a}{b} \Theta_{a b}=\lim _{\alpha \rightarrow 0^{+}} \int_{-i \infty}^{+i \infty} \frac{\mathrm{d} \lambda}{2 \pi i} \frac{1}{(\lambda+\alpha)^{2}}\left(\frac{a}{b}\right)^{\lambda} \equiv \int \mathrm{d}[\lambda]\left(\frac{a}{b}\right)^{\lambda}
$$

valid for $a, b>0$, which allows us to write

$$
\begin{aligned}
I_{m} & =\frac{A_{\varepsilon}}{2} \int \mathrm{d}[\lambda]\left(m^{2}\right)^{\lambda} \int \frac{\mathrm{d}\left[\boldsymbol{k}_{1}\right]}{\boldsymbol{q}^{2}\left(\boldsymbol{k}_{1}^{2}\right)^{1+\lambda}} \\
& =\frac{A_{\varepsilon}}{2} \int \mathrm{d}[\lambda] \frac{\Gamma(1+\lambda-\varepsilon) \Gamma(\varepsilon) \Gamma(\varepsilon-\lambda)}{\Gamma(1+\lambda) \Gamma(2 \varepsilon-\lambda)}\left(m^{2}\right)^{\lambda}\left(\boldsymbol{k}_{2}^{2}\right)^{-1-\lambda+\varepsilon} .
\end{aligned}
$$

The integrand vanishes for $|\lambda| \rightarrow \infty$ in all directions apart from the real axis. As before, we consider first the case $\boldsymbol{k}_{2}^{2}>m^{2}$ and displace the integration contour around the positive real semiaxis enclosing all the poles placed in $\lambda>0$. The smaller pole, at $\lambda=\varepsilon$, gives us the result at order $\mathcal{O}\left(m / k_{2}\right)$

$$
I_{m}=\bar{\alpha}_{s} h_{\mathrm{q}}^{(0)}\left(\boldsymbol{k}_{2}\right)\left\{\frac{1}{2 \varepsilon^{2} \Gamma(1-\varepsilon) \Gamma(1+\varepsilon)}\left(\frac{m^{2}}{\mu^{2}}\right)^{\varepsilon}+\mathcal{O}\left(m / k_{2}\right)\right\} .
$$


On the other hand, for $\boldsymbol{k}_{2}^{2}<m^{2}$, we consider the poles placed at the negative real semiaxis and therefore the residue at $\lambda=-\alpha$ with $\alpha \rightarrow 0^{+}$. By taking into account also the virtual contribution to $K_{0}$ in this case, we obtain

$$
\begin{aligned}
I_{m} & -h_{\mathrm{q}}^{(0)}\left(\boldsymbol{k}_{2}\right) \omega^{(1)}\left(\boldsymbol{k}_{2}^{2}\right) \log \left(\frac{\boldsymbol{k}_{2}^{2}}{m^{2}}\right)=h_{\mathrm{q}}^{(0)}\left(\boldsymbol{k}_{2}\right)\left\{\omega ^ { ( 1 ) } ( \boldsymbol { k } _ { 2 } ^ { 2 } ) \left[\log \left(\frac{\boldsymbol{k}_{2}^{2}}{m^{2}}\right)\right.\right. \\
& \left.+2[\psi(1)-\psi(1-\varepsilon)+\psi(\varepsilon)-\psi(2 \varepsilon)]]+\mathcal{O}\left(k_{2} / m\right)\right\} .
\end{aligned}
$$

Eq.(4.8) and eq.(4.9) show double log singularities also.

\subsection{Impact factors}

Finally, summing up all the pieces according to eq.(3.16), the impact factor for heavy quarks at the next-to-leading level can be written as

$$
h_{\mathbf{q}}\left(\boldsymbol{k}_{2}\right)=h_{\mathbf{q}}^{(0)}\left(\boldsymbol{k}_{2}\right)+h_{\mathbf{q}}^{(1)}\left(\boldsymbol{k}_{2}\right),
$$

where

$$
\begin{aligned}
& h_{\mathrm{q}}^{(1)}\left(\boldsymbol{k}_{2}\right)=h_{\mathbf{q}, m=0}^{(1)}\left(\boldsymbol{k}_{2}\right)+h_{\mathrm{q}}^{(0)}\left(\boldsymbol{k}_{2}\right) \\
\times & \left\{\omega^{(1)}\left(m^{2}\right) \frac{\Gamma(1+2 \varepsilon)}{\varepsilon \Gamma^{2}(1+\varepsilon)}\left[\frac{2+3 \varepsilon+2 \varepsilon^{2}}{2(1+2 \varepsilon)}-\frac{1}{\Gamma(1-\varepsilon) \Gamma(1+\varepsilon)}\right]+\mathcal{O}\left(m / k_{2}\right)\right\},
\end{aligned}
$$

is valid in the limit $\boldsymbol{k}_{2}^{2}>m^{2}$, and

$$
\begin{aligned}
& h_{\mathbf{q}}^{(1)}\left(\boldsymbol{k}_{2}\right)=h_{\mathbf{q}, m=0}^{(1)}\left(\boldsymbol{k}_{2}\right)+h_{\mathbf{q}}^{(0)}\left(\boldsymbol{k}_{2}\right) \\
\times & \left\{\omega^{(1)}\left(\boldsymbol{k}_{2}^{2}\right)\left[\psi(1)-\psi(1-\varepsilon)-\frac{1+5 \varepsilon-2 \varepsilon^{2}}{2(1+2 \varepsilon)}\right]+\mathcal{O}\left(k_{2} / m\right)\right\},
\end{aligned}
$$

is valid for $\boldsymbol{k}_{2}^{2}<m^{2}$.

We notice, in the first place, that all double $\log$ contributions of type $1 / \varepsilon^{2}$ and $1 / \varepsilon \log \left(\boldsymbol{k}_{2}^{2} / m^{2}\right)$ appearing in eqs. 4.4 4.5) and 4.84.9) have canceled out in eqs. (4.11) and (4.12). This means that indeed our subtraction of the leading kernel was effective, thus lending credit to the scale (3.9) and to the $H$-kernel (3.13).

The remaining singularities of the impact factor are single logarithmic ones $\sim 1 / \varepsilon$, having the structure

$$
\left.h_{\mathbf{q}}^{(1)}\left(\boldsymbol{k}_{2}\right)\right|_{s i n g}=h_{\mathbf{q}}^{(0)}\left(\boldsymbol{k}_{2}\right)\left(\frac{3}{2} \omega^{(1)}\left(\boldsymbol{k}_{2}^{2}\right)-\frac{1}{2} \omega^{(1)}\left(m^{2}\right) \Theta_{k_{2} m}-\frac{1}{2} \omega^{(1)}\left(\boldsymbol{k}_{2}^{2}\right) \Theta_{m k_{2}}\right) \text {. }
$$

Here the first term has the customary collinear interpretation [4], as coming from the finite part of the $\mathrm{q} \rightarrow \mathrm{g}$ anomalous dimension

$$
\gamma_{\mathrm{gq}}-\frac{C_{F} \alpha_{s}}{\pi \omega}=-\frac{C_{F} \alpha_{s}}{2 \pi}\left(\frac{3}{2}-\frac{1}{2} \varepsilon\right)
$$


while the remaining ones - depending on the scale $\operatorname{Min}\left(k_{2}, m\right)$ - do not have such interpretation. Note, however, that a finite mass scale change $m \rightarrow c m$, will produce exactly this type of contributions from the singular integration of the $K_{0}$ kernel in eq. (3.10) acting on $h_{\mathrm{q}}^{(0)}\left(\boldsymbol{k}_{1}\right)$ over the region $0<k_{1}<\operatorname{Min}\left(k_{2}, m\right)$, leading to the expression

$$
\delta h_{a}^{(1)}\left(\boldsymbol{k}_{2}\right)=h_{\mathbf{q}}^{(0)}\left(\boldsymbol{k}_{2}\right) a(c)\left[\omega^{(1)}\left(m^{2}\right) \Theta_{k_{2} m}+\omega^{(1)}\left(\boldsymbol{k}_{2}^{2}\right) \Theta_{m k_{2}}\right] .
$$

Therefore, the singularities (4.13) can finally be interpreted in the form

$$
\begin{aligned}
\left.h_{\mathbf{q}}^{(1)}\left(\boldsymbol{k}_{2}\right)\right|_{s i n g} & =h_{\mathbf{q}}^{(0)}\left(\boldsymbol{k}_{2}\right) \frac{3}{2}\left[\omega^{(1)}\left(\boldsymbol{k}_{2}^{2}\right)-\omega^{(1)}\left(m^{2}\right)\right] \Theta_{k_{2} m}+\delta h_{1}^{(1)}\left(\boldsymbol{k}_{2}\right) \\
& =h_{\mathbf{q}}^{(0)}\left(\boldsymbol{k}_{2}\right) \frac{\alpha_{s} N_{c}}{2 \pi}\left(-\frac{3}{2} \log \frac{\boldsymbol{k}_{2}^{2}}{m^{2}}\right) \Theta_{k_{2} m}+\delta h_{1}^{(1)}\left(\boldsymbol{k}_{2}\right),
\end{aligned}
$$

meaning that $h_{\mathrm{q}}^{(1)}$ is actually finite, with the $\log \left(\boldsymbol{k}_{2}^{2} / \mathrm{m}^{2}\right)$ dependence predicted by the DGLAP equations, apart from a proper mass scale change in eq.(3.13). In other words, the scale leading to a finite massive quark impact factor differs from eq. (3.9) by a finite renormalization of the quark mass, which is a normal ambiguity in this type of problems.

Our final result for the heavy quark impact factor at the next-to-leading level reads

$$
h_{\mathrm{q}}\left(\boldsymbol{k}_{2}\right)=\left.h_{\mathrm{q}}^{(1)}\left(\boldsymbol{k}_{2}\right)\right|_{\text {sing }}+\left.h_{\mathrm{q}}\left(\boldsymbol{k}_{2}\right)\right|_{\text {finite }},
$$

where the singular piece is defined in eq.(4.16) and

$$
\begin{aligned}
\left.h_{\mathrm{q}}\left(\boldsymbol{k}_{2}\right)\right|_{\text {finite }} & =h_{\mathrm{q}}^{(0)}\left(\alpha_{s}\left(\boldsymbol{k}_{2}\right)\right) \\
\times & \left\{1+\frac{\alpha_{s} N_{c}}{2 \pi}\left[\mathcal{K}-\frac{\pi^{2}}{6}-\left(\frac{3}{2}+\sum_{\operatorname{Re} \gamma>1} \operatorname{Res}[\tilde{h}(\gamma)]\right) \Theta_{k_{2} m}\right.\right. \\
& \left.\left.+\left(2+\sum_{\operatorname{Re\gamma }<1} \operatorname{Res}[\tilde{h}(\gamma)]\right) \Theta_{m k_{2}}\right]\right\}
\end{aligned}
$$

is the finite contribution to the heavy quark impact factor, with

$$
\mathcal{K}=\frac{67}{18}-\frac{\pi^{2}}{6}-\frac{5 n_{f}}{9 N_{c}}
$$

the constant term of the impact factor for massless quarks, eq.(3.14), and

$$
\begin{aligned}
\tilde{h}(\gamma) & =\left(\frac{\boldsymbol{k}_{2}^{2}}{m^{2}}\right)^{1-\gamma}\left\{\frac{\Gamma(\gamma) \Gamma^{3}(1-\gamma)}{4 \Gamma(2-2 \gamma)}\left[\frac{1}{\gamma}+\frac{2}{1-2 \gamma}\left(\frac{1}{1-\gamma}-\frac{1}{3-2 \gamma}\right)\right]\right. \\
& \left.-\frac{1}{(1-\gamma)^{2}}[\psi(1-\gamma)+\psi(\gamma)-2 \psi(1)]\right\} .
\end{aligned}
$$


The compact expression (4.20) was obtained by adding to the Mellin transform (4.2) the contribution of eq.(4.7), with $\lambda \rightarrow-1+\gamma+2 \varepsilon$, and the Mellin transform of the virtual piece of the last term at the r.h.s of eq.(3.16). The sum of these three terms, apart from $\gamma=1-\varepsilon, 1-2 \varepsilon$, whose contributions have already been treated separately, is finite and therefore was expanded for $\varepsilon \rightarrow 0$. As for the massless case, the singularities proportional to $\left(11 / 6-n_{f} / 3 N_{c}\right)$, the beta function, were absorbed by the running strong coupling constant $\alpha_{s}\left(\boldsymbol{k}_{2}\right)$. The function $\tilde{h}(\gamma)$ provides the corrections of order $\mathcal{O}\left(m / k_{2}\right)$ and $\mathcal{O}\left(k_{2} / m\right)$ to the impact factor for $m^{2}<\boldsymbol{k}_{2}^{2}$ and $m^{2}>\boldsymbol{k}_{2}^{2}$ respectively, yielding the following final result

$$
\begin{aligned}
& \sum_{\operatorname{Re} \gamma>1} \operatorname{Res}[\tilde{h}(\gamma)]=L i_{2}\left(\frac{m^{2}}{\boldsymbol{k}_{2}^{2}}\right)+\sum_{n=1}^{\infty} \frac{\Gamma(2 n+2)}{\Gamma^{2}(n+1)}\left(-\frac{m^{2}}{\boldsymbol{k}_{2}^{2}}\right)^{n} \\
& \times\left\{\frac{1}{n^{2}}-\frac{1}{2(n+1)^{2}}-\frac{1}{2(2 n-1)^{2}}-\frac{6}{(2 n+1)^{3}}-\frac{3}{2(2 n+1)^{2}}\right. \\
& \quad+\left(\frac{2}{n}-\frac{1}{n+1}-\frac{1}{2(2 n-1)}-\frac{3}{(2 n+1)^{2}}-\frac{3}{2(2 n+1)}\right) \\
&\left.\quad \times\left(\psi(n+1)-\psi(2 n+2)-\frac{1}{2} \log \frac{m^{2}}{\boldsymbol{k}_{2}^{2}}\right)\right\}
\end{aligned}
$$

and

$$
\begin{aligned}
& \sum_{\operatorname{Re} \gamma<1} \operatorname{Res}[\tilde{h}(\gamma)]=L i_{2}\left(\frac{\boldsymbol{k}_{2}^{2}}{m^{2}}\right)-\frac{3 \pi^{2}}{8} \sqrt{\frac{\boldsymbol{k}_{2}^{2}}{m^{2}}}+\frac{\boldsymbol{k}_{2}^{2}}{m^{2}}\left(\frac{5}{6}-\frac{1}{4} \log \frac{\boldsymbol{k}_{2}^{2}}{m^{2}}\right) \\
& +\sum_{n=1}^{\infty} \frac{\Gamma^{2}(n+1)}{\Gamma(2 n+2)}\left(-\frac{\boldsymbol{k}_{2}^{2}}{m^{2}}\right)^{n+1}\left(\frac{1}{n}+\frac{2}{n+1}-\frac{3}{2 n+1}-\frac{1}{2 n+3}\right) .
\end{aligned}
$$

\section{Conclusions}

Starting from the explicit squared matrix element for gluon emission in eq.(3.1) we have motivated the subtraction of the leading term in eq.(3.8), and we have performed the $\boldsymbol{k}_{1}$ and $z_{1}$ integrals needed to provide an explicit result for the heavy quark impact factor in eq.(4.16) and eq.(4.18).

Even if the cross section being investigated is unphysical, the relevance of our results stems from the consistency of the following features: (i) the validity of the $\boldsymbol{k}$-factorization formula (2.1) with scale $s_{0}=\operatorname{Max}\left(k_{1}, m_{1}\right) \operatorname{Max}\left(k_{2}, m_{2}\right)$; (ii) the explicit expression of the impact factor with factorizable single logarithmic collinear divergences, and (iii) the probe-independence of the subleading $H$-kernels of the CC scheme [四], defined in eq.(3.13).

Even if such universal extra kernels can be reabsorbed in the impact factors for colourless sources [6], they help clarifying the structure of the collinear limits for two-scale processes as elaborated at length by CCS [1]. Here, it was shown that the 
gluonic Green's function including such kernels, is free of double logs of collinear type for both $k_{1} \gg k_{2}$ and $k_{2} \gg k_{1}$. As a consequence, even colourless impact factors will show, in the present scheme, simple collinear properties, as expected from their DGLAP analysis [8].

Of course, the real problem is to provide an explicit expression for the DIS impact factors. But - if the lesson learned form the L and NL kernels is still valid - the impact factor's magnitude is not expected to be much different from their approximate collinear evaluation.

\section{Aknowledgements}

We wish to thank Gianni Camici for very valuable contributions in the early stages of this paper. Work supported in part by the E.U. QCDNET contract FMRX-CT980194 and by MURST (Italy). G.R. acknowledges financial support from INFN (Italy) and BMBF Project 05HT9VKB0 (Germany).

\section{A. Real contribution to the fragmentation vertex}

The differential cross section for real gluon emission off a heavy quark, eq.(3.1), can be simplified by using identities of the following type

$$
\begin{aligned}
& \frac{z_{1}^{3}\left(1-z_{1}\right)\left[\left(2 \boldsymbol{q}-z_{1} \boldsymbol{k}_{2}\right) \cdot \boldsymbol{k}_{2}\right]^{2}}{\left[\boldsymbol{q}^{2}+m^{2} z_{1}^{2}\right]^{2}\left[\left(\boldsymbol{q}-z_{1} \boldsymbol{k}_{2}\right)^{2}+m^{2} z_{1}^{2}\right]^{2}}=z_{1}\left(1-z_{1}\right)\left\{\frac{1}{\left[\boldsymbol{q}^{2}+m^{2} z_{1}^{2}\right]^{2}}\right. \\
& \left.+\frac{1}{\left[\left(\boldsymbol{q}-z_{1} \boldsymbol{k}_{2}\right)^{2}+m^{2} z_{1}^{2}\right]^{2}}-\frac{2}{\left[\boldsymbol{q}^{2}+m^{2} z_{1}^{2}\right]\left[\left(\boldsymbol{q}-z_{1} \boldsymbol{k}_{2}\right)^{2}+m^{2} z_{1}^{2}\right]}\right\},
\end{aligned}
$$

to split the full expression into several contributions with at most two different propagators free of $\boldsymbol{q}$ dependences at the numerator. After some algebra, we obtain the

following expression for the real contribution to the fragmentation vertex of quark $q$

$$
\begin{aligned}
F_{\mathbf{q}, \text { real }}\left(z_{1}, \boldsymbol{k}_{1}, \boldsymbol{k}_{2}\right)=A_{\varepsilon}\left\{-z_{1}\left(1-z_{1}\right) \frac{m^{2}}{\boldsymbol{k}_{2}^{2}}\left[\frac { C _ { F } } { N _ { c } } \left(\frac{1}{\left[\boldsymbol{q}^{2}+m^{2} z_{1}^{2}\right]^{2}}\right.\right.\right. \\
\left.\left.+\frac{1}{\left[\left(\boldsymbol{q}-z_{1} \boldsymbol{k}_{2}\right)^{2}+m^{2} z_{1}^{2}\right]^{2}}\right)+\frac{1}{\left[\boldsymbol{k}_{1}^{2}+m^{2} z_{1}^{2}\right]^{2}}\right] \\
+\left(\frac{C_{F}}{N_{c}}-\frac{1}{2}\right) \frac{z_{1}^{2} \mathcal{P}_{\mathbf{g q}}\left(z_{1}, \varepsilon\right)+2 z_{1}\left(1-z_{1}\right)\left(m^{2} / \boldsymbol{k}_{2}^{2}\right)}{\left[\boldsymbol{q}^{2}+m^{2} z_{1}^{2}\right]\left[\left(\boldsymbol{q}-z_{1} \boldsymbol{k}_{2}\right)^{2}+m^{2} z_{1}^{2}\right]} \\
+\frac{\mathcal{P}_{\mathbf{g q}}\left(z_{1}, \varepsilon\right)+2 z_{1}\left(1-z_{1}\right)\left(m^{2} / \boldsymbol{k}_{2}^{2}\right)}{2\left[\boldsymbol{k}_{1}^{2}+m^{2} z_{1}^{2}\right]\left[\boldsymbol{q}^{2}+m^{2} z_{1}^{2}\right]} \\
\left.+\frac{\left(1-z_{1}\right)^{2} \mathcal{P}_{\mathbf{g q}}\left(z_{1}, \varepsilon\right)+2 z_{1}\left(1-z_{1}\right)\left(m^{2} / \boldsymbol{k}_{2}^{2}\right)}{2\left[\boldsymbol{k}_{1}^{2}+m^{2} z_{1}^{2}\right]\left[\left(\boldsymbol{q}-z_{1} \boldsymbol{k}_{2}\right)^{2}+m^{2} z_{1}^{2}\right]}\right\} .
\end{aligned}
$$


According to eq. (3.10), this expression has to be integrated first in $\boldsymbol{k}_{1}$ and then in $z_{1}$, for $z_{1}>q / \sqrt{s}$. To perform the first integration we use

$$
\begin{aligned}
& \int \frac{\mathrm{d}\left[\boldsymbol{k}_{1}\right]}{\left[\boldsymbol{k}_{1}^{2}+m^{2} z_{1}^{2}\right]^{\alpha}\left[\left(\boldsymbol{k}_{1}+\boldsymbol{p}\right)^{2}+m^{2} z_{1}^{2}\right]^{\beta}}= \\
& \frac{\Gamma(\alpha+\beta-1-\varepsilon)}{\Gamma(\alpha) \Gamma(\beta)} \int_{0}^{1} d x \frac{x^{\alpha-1}(1-x)^{\beta-1}}{\left[x(1-x) p^{2}+m^{2} z_{1}^{2}\right]^{\alpha+\beta-1-\varepsilon}},
\end{aligned}
$$

then, we obtain

$$
\begin{aligned}
& F_{\mathbf{q}, \text { real }}\left(z_{1}, \boldsymbol{k}_{2}\right)=\int \mathrm{d}\left[\boldsymbol{k}_{1}\right] F_{\mathbf{q}, \text { real }}\left(z_{1}, \boldsymbol{k}_{1}, \boldsymbol{k}_{2}\right) \\
& =A_{\varepsilon} \Gamma(1-\varepsilon)\left\{-\left(\frac{2 C_{F}}{N_{c}}+1\right) z_{1}^{-1+2 \varepsilon}\left(1-z_{1}\right) \frac{\left(m^{2}\right)^{\varepsilon}}{\boldsymbol{k}_{2}^{2}}\right. \\
& +\int_{0}^{1} \mathrm{~d} x\left[\left(\frac{C_{F}}{N_{c}}-\frac{1}{2}\right) \frac{z_{1}^{2 \varepsilon} \mathcal{P}_{\mathrm{gq}}\left(z_{1}, \varepsilon\right)+2 z_{1}^{-1+2 \varepsilon}\left(1-z_{1}\right)\left(m^{2} / \boldsymbol{k}_{2}^{2}\right)}{\left[x(1-x) \boldsymbol{k}_{2}^{2}+m^{2}\right]^{1-\varepsilon}}\right. \\
& +\frac{\mathcal{P}_{\mathrm{gq}}\left(z_{1}, \varepsilon\right)+2 z_{1}\left(1-z_{1}\right)\left(m^{2} / \boldsymbol{k}_{2}^{2}\right)}{2\left[x(1-x) \boldsymbol{k}_{2}^{2}+m^{2} z_{1}^{2}\right]^{1-\varepsilon}} \\
& +\frac{\left(1-z_{1}\right)^{2} \mathcal{P}_{\mathrm{gq}}\left(z_{1}, \varepsilon\right)+2 z_{1}\left(1-z_{1}\right)\left(m^{2} / \boldsymbol{k}_{2}^{2}\right)}{\left.\left.2\left[x(1-x)\left(1-z_{1}\right)^{2} \boldsymbol{k}_{2}^{2}+m^{2} z_{1}^{2}\right]^{1-\varepsilon}\right]\right\}} .
\end{aligned}
$$

By subtracting the massless contribution

$$
\Delta F_{\mathbf{q}, \text { real }}\left(z_{1}, \boldsymbol{k}_{2}\right)=F_{\mathbf{q}, \text { real }}\left(z_{1}, \boldsymbol{k}_{2}\right)-F_{\mathbf{q}, \text { real }}^{m=0}\left(z_{1}, \boldsymbol{k}_{2}\right)
$$

we get an expression that is regular at $z_{1}=0$ and therefore can be integrated down 
to $z_{1}=0$ without changing the final result. Let's define

$$
\begin{aligned}
& \Delta F_{\mathbf{q}, \text { real }}\left(\boldsymbol{k}_{2}\right)=\int_{0}^{1} \mathrm{~d} z_{1} \Delta F_{\mathbf{q}, \text { real }}\left(z_{1}, \boldsymbol{k}_{2}\right) \\
& =A_{\varepsilon}\left[\left(\frac{C_{F}}{N_{c}}+\frac{1}{2}\right) \frac{\Gamma(-\varepsilon)}{1+2 \varepsilon} \frac{\left(m^{2}\right)^{\varepsilon}}{\boldsymbol{k}_{2}^{2}}\right. \\
& -\left(\frac{C_{F}}{N_{c}}-\frac{1}{2}\right) \frac{\Gamma(-\varepsilon)}{2}\left\{\left(\frac{1}{1+2 \varepsilon}+\frac{\varepsilon}{2}\right)\right. \\
& \times \int_{0}^{1} \mathrm{~d} x\left[\frac{1}{\left[x(1-x) \boldsymbol{k}_{2}^{2}+m^{2}\right]^{1-\varepsilon}}-\frac{1}{\left[x(1-x) \boldsymbol{k}_{2}^{2}\right]^{1-\varepsilon}}\right] \\
& \left.+\frac{2\left(m^{2} / \boldsymbol{k}_{2}^{2}\right)}{1+2 \varepsilon} \int_{0}^{1} \frac{\mathrm{d} x}{\left[x(1-x) \boldsymbol{k}_{2}^{2}+m^{2}\right]^{1-\varepsilon}}\right\} \\
& +\frac{\Gamma(1-\varepsilon)}{2}\left\{\int_{0}^{1} \int_{0}^{1} \mathrm{~d} z_{1} \mathrm{~d} x\left(\frac{1-z_{1}}{z_{1}}+\frac{1+\varepsilon}{2} z_{1}\right)\right. \\
& \times\left[\frac{1}{\left[x(1-x) \boldsymbol{k}_{2}^{2}+m^{2} z_{1}^{2}\right]^{1-\varepsilon}}-\frac{1}{\left[x(1-x) \boldsymbol{k}_{2}^{2}\right]^{1-\varepsilon}}\right. \\
& \left.+\frac{\left(1-z_{1}\right)^{2}}{\left[x(1-x)\left(1-z_{1}\right)^{2} \boldsymbol{k}_{2}^{2}+m^{2} z_{1}^{2}\right]^{1-\varepsilon}}-\frac{\left(1-z_{1}\right)^{2}}{\left[x(1-x)\left(1-z_{1}\right)^{2} \boldsymbol{k}_{2}^{2}\right]^{1-\varepsilon}}\right] \\
& +\frac{2 m^{2}}{\boldsymbol{k}_{2}^{2}} \int_{0}^{1} \int_{0}^{1} z_{1}\left(1-z_{1}\right) \mathrm{d} z_{1} \mathrm{~d} x\left[\frac{1}{\left[x(1-x) \boldsymbol{k}_{2}^{2}+m^{2} z_{1}^{2}\right]^{1-\varepsilon}}\right. \\
& \left.\left.\left.+\frac{1}{\left[x(1-x)\left(1-z_{1}\right)^{2} \boldsymbol{k}_{2}^{2}+m^{2} z_{1}^{2}\right]^{1-\varepsilon}}\right]\right\}\right] \text {. }
\end{aligned}
$$

Notice that some of the integrations has been kept undone. This long expression, although cumbersome, will be drastically simplified after adding the virtual contribution before doing any further integration.

\section{B. Virtual contribution to the fragmentation vertex}

The correction to the cross section due to virtual emission, including subleading effects, for general parton-parton scattering, can be extracted from the amplitude of Ref. [7] $\left(t=-\boldsymbol{k}_{1}^{2}\right)$

$$
\begin{aligned}
\mathcal{M}_{\mathrm{ab}} & =2 s g^{2}\left(\boldsymbol{t}_{\mathrm{a}}^{c} \boldsymbol{t}_{\mathrm{b}}^{c}\right)\left[\delta_{\lambda_{3}, \lambda_{1}}\left(1+\Gamma_{\mathrm{aa}}^{(+)}\right)+\delta_{\lambda_{3},-\lambda_{1}} \Gamma_{\mathrm{aa}}^{(-)}\right] \frac{1}{t}\left[1+\omega(-t) \log \frac{s}{-t}\right] \\
& \times\left[\left(1+\Gamma_{\mathrm{bb}}^{(+)}\right) \delta_{\lambda_{4}, \lambda_{2}}+\Gamma_{\mathrm{bb}}^{(-)} \delta_{\lambda_{4},-\lambda_{2}}\right]
\end{aligned}
$$

were $\Gamma^{(+)}$and $\Gamma^{(-)}$are the helicity conserving and the helicity non-conserving con-

tributions respectively. At the order we are working, the virtual terms contribute to 
the fragmentation vertex as follows

$$
F_{\mathbf{q}, v i r t}\left(z_{1}, \boldsymbol{k}_{1}, \boldsymbol{k}_{2}\right)=h_{\mathbf{q}}^{(0)}\left(\boldsymbol{k}_{1}\right) 2 \Gamma_{\mathbf{q q}}^{(+)}\left(\boldsymbol{k}_{1}\right) \delta\left(1-z_{1}\right) \delta[\boldsymbol{q}] .
$$

As for real emission, we split $\Gamma_{\mathrm{qq}}^{(+)}$into a massless contribution plus a quark mass dependent extra term

$$
\Gamma_{\mathrm{qq}}^{(+)}=\Gamma_{\mathrm{qq}, m=0}^{(+)}+\Delta \Gamma_{\mathrm{qq}}^{(+)},
$$

where $\Delta \Gamma_{\mathrm{qq}}^{(+)}$cancels for $m=0$, being

$$
\begin{aligned}
\Gamma_{\mathrm{qq}, m=0}^{(+)}(\boldsymbol{k}) & =\frac{\omega^{(1)}\left(\boldsymbol{k}^{2}\right)}{2}\{\psi(1-\varepsilon)-2 \psi(\varepsilon)+\psi(1) \\
& +\frac{1}{1+2 \varepsilon}\left(\frac{1}{4(3+2 \varepsilon)}-\frac{2}{\varepsilon}-\frac{7}{4}-\frac{n_{f}}{N_{c}} \frac{1+\varepsilon}{3+2 \varepsilon}\right)-\frac{1}{2} \\
& \left.+\frac{2}{\varepsilon} \frac{C_{F}}{N_{c}}\left(\frac{1}{1+2 \varepsilon}+\frac{\varepsilon}{2}\right)\right\},
\end{aligned}
$$

and

$$
\begin{aligned}
\Delta \Gamma_{\mathrm{qq}}^{(+)} & (\boldsymbol{k})=\frac{\bar{\alpha}_{s}}{4 \Gamma(1-\varepsilon) \mu^{2 \varepsilon}}\left[\Gamma ( - \varepsilon ) ( \frac { C _ { F } } { N _ { c } } - \frac { 1 } { 2 } ) \left\{\left(\frac{1}{1+2 \varepsilon}+\frac{\varepsilon}{2}\right)\right.\right. \\
& \times \int_{0}^{1} \mathrm{~d} x\left[\frac{1}{\left[x(1-x) \boldsymbol{k}^{2}+m^{2}\right]^{1-\varepsilon}}-\frac{1}{\left[x(1-x) \boldsymbol{k}^{2}\right]^{1-\varepsilon}}\right] \\
& \left.+\frac{2\left(m^{2} / \boldsymbol{k}^{2}\right)}{1+2 \varepsilon} \int_{0}^{1} \frac{\mathrm{d} x}{\left[x(1-x) \boldsymbol{k}^{2}+m^{2}\right]^{1-\varepsilon}}\right\}-\frac{C_{F}}{N_{c}} \frac{2 \Gamma(-\varepsilon)}{1+2 \varepsilon} \frac{\left(m^{2}\right)^{\varepsilon}}{\boldsymbol{k}^{2}} \\
& -\Gamma(1-\varepsilon)\left\{\int_{0}^{1} \int_{0}^{1} \mathrm{~d} x \mathrm{~d} y(1-x)^{2}\left(\frac{1-x}{x}+\frac{1+\varepsilon}{2} x\right)\right. \\
& \times\left[\frac{1}{\left[y(1-y)(1-x)^{2} \boldsymbol{k}^{2}+m^{2} x^{2}\right]^{1-\varepsilon}}-\frac{1}{\left[y(1-y)(1-x)^{2} \boldsymbol{k}^{2}\right]^{1-\varepsilon}}\right] \\
+ & \left.\left.\frac{2 m^{2}}{\boldsymbol{k}^{2}} \int_{0}^{1} \int_{0}^{1} \frac{x(1-x) \mathrm{d} x \mathrm{~d} y}{\left[y(1-y)(1-x)^{2} \boldsymbol{k}^{2}+m^{2} x^{2}\right]^{1-\varepsilon}}\right\}\right]
\end{aligned}
$$

We define

$$
\begin{aligned}
\Delta F_{\mathrm{q}, v i r t}\left(\boldsymbol{k}_{2}\right) & =\int_{0}^{1} \mathrm{~d} z_{1} \int \mathrm{d}\left[\boldsymbol{k}_{1}\right] \Delta F_{\mathrm{q}, v i r t}\left(z_{1}, \boldsymbol{k}_{1}, \boldsymbol{k}_{2}\right) \\
& =h_{\mathbf{q}}^{(0)}\left(\boldsymbol{k}_{2}\right) 2 \Delta \Gamma_{\mathrm{qq}}^{(+)}\left(\boldsymbol{k}_{2}\right) .
\end{aligned}
$$

Adding up together the real, eq.(A.6), and the virtual, eq.(B.6), contributions to $\Delta F_{\mathrm{q}}\left(\boldsymbol{k}_{2}\right)$ most of the terms cancel. To see this cancellation it is enough to identify the integration variable $x$ appearing in $\Delta \Gamma_{\mathrm{qq}}^{(+)}\left(\boldsymbol{k}_{2}\right)$, eq.(B.5), with the momentum fraction $z_{1}$ appearing in eq.(A.6). In particular, the $C_{F}$ contribution fully cancels and the $N_{c}$ part gives the the simplified result presented in eq.(4.1). 


\section{References}

[1] G.P. Salam, JHEP 9807(1998)019, hep-ph/9806482; M. Ciafaloni and D. Colferai, Phys. Lett. B 452 (1999) 372, hep-ph/9812366; M. Ciafaloni, D. Colferai and G.P. Salam, Phys. Rev. D 60 (1999) 114036, hep-ph/9905566.

[2] V.S. Fadin and L.N. Lipatov, Phys. Lett. B 429 (1998) 127, hep-ph/9802290; M. Ciafaloni and G. Camici, Phys. Lett. B 430 (1998) 249, hep-ph/9803389.

[3] M. Ciafaloni, Phys. Lett. B 429 (1998) 363, hep-ph/9801322.

[4] M. Ciafaloni and D. Colferai, Nucl. Phys. B 538 (1999) 187, hep-ph/9806350.

[5] V.S. Fadin, R. Fiore, M.I. Kotsky and A. Papa, hep-ph/9908265.

[6] V.S. Fadin and A.D. Martin, Phys. Rev. D 60 (1999) 114008, hep-ph/9904505.

[7] V.S. Fadin and L.N. Lipatov, Nucl. Phys. B 406 (1993) 259; V.S. Fadin, R. Fiore and A. Quartarolo, Phys. Rev. D 50 (1994) 2265; Phys Rev. D 50 (1994) 5893; V.S. Fadin, R. Fiore and M.I. Kotsky, Phys. Lett. B 359 (1995) 181; B 387 (1996) 593; B 389 (1996) 737.

[8] M. Taiuti, University of Florence Thesis (February 2000), unpublished. 